\title{
Malignant fibrous histiocytoma mimicking pulmonary embolism
}

\author{
T J W SLEYSTER, F M J HEYSTRATEN \\ From the Department of Radiodiagnosis, Catholic University Hospital, Nijmegen, The Netherlands
}

ABSTRACT A rare case of malignant fibrous histiocytoma occurring in the pulmonary artery is reported. Such primary pulmonary artery sarcomas may have the diagnosis suggested by angiography or echocardiography; radiographically and in perfusionventilation scanning their usual unilateral origin (with later spread) and the persistence of the perfusion defect are among the features normally distinguishing them from thromboembolism.

Primary neoplasms originating from the intima of the major blood vessels are rare. Malignant fibrous histiocytoma is a pleomorphic mesenchymal sarcoma, whose most common primary sites are the extremities and retroperitoneum, and only very rarely the aorta and pulmonary artery. Pulmonary artery sarcomas, including malignant fibrous histiocytoma, present most often with symptoms of acute and recurrent pulmonary embolism. We report a case of malignant fibrous histiocytoma occurring in the pulmonary artery.

\section{Case report}

A 45 year old man presented with symptoms of pulmonary embolism. There was no evidence of deep vein thrombosis. The erythrocyte sedimentation rate was $61 \mathrm{~mm}$ in one hour. A chest radiograph showed decreased vascular markings on the left side. A ventilation-perfusion scan showed absence of perfusion of the whole of the left lung with normal ventilation. An echocardiogram was normal. An angiogram showed occlusion of the left main pulmonary artery.

A large pulmonary embolus was diagnosed, and the patient was given anticoagulants and discharged symptom free one week later. Almost one year later the patient presented with an episode of haemoptysis and with progressive dyspnoea. A chest radiograph showed a left hilar mass. An angiogram showed that the left pulmonary artery was still totally occluded, with retrograde growth of an intraluminal mass and in addition a large lobulated mural mass almost occluding the right main pulmonary artery with multiple peripheral perfusion defects (figure, $\mathbf{A}$ and $\mathrm{B}$ ).

Bronchoscopy showed a raised lesion in the left upper lobe bronchus. After a mediastinoscopy, in which no mass was

Address for reprint requests: Dr F M J Heysraten, Department of Radiodiagnosis, Catholic University Hospital St Radboud, Geert Grooteplein Zuid 18, 6525 GA Nijmegen, The Netherlands.

Accepted 7 January 1988 seen, the pulmonary artery was explored. Intraluminalo myxomatous tissue was found which was adhering only to $\overrightarrow{\vec{f}}$ the vessel wall on the left side. Microscopic examination of the bronchoscopic and pulmonary tissue revealed malignant $\vec{\omega}$ fibrous histiocytoma (pleomorphic type). Owing to the poor. condition of the patient no further treatment was offered. Two months later he died of respiratory and cardiac failure. At necropsy a tumour was found to have affected the pulmonary valve and parietal pleura on the left side inaddition to the pulmonary artery. No tumour was found outside the thorax.

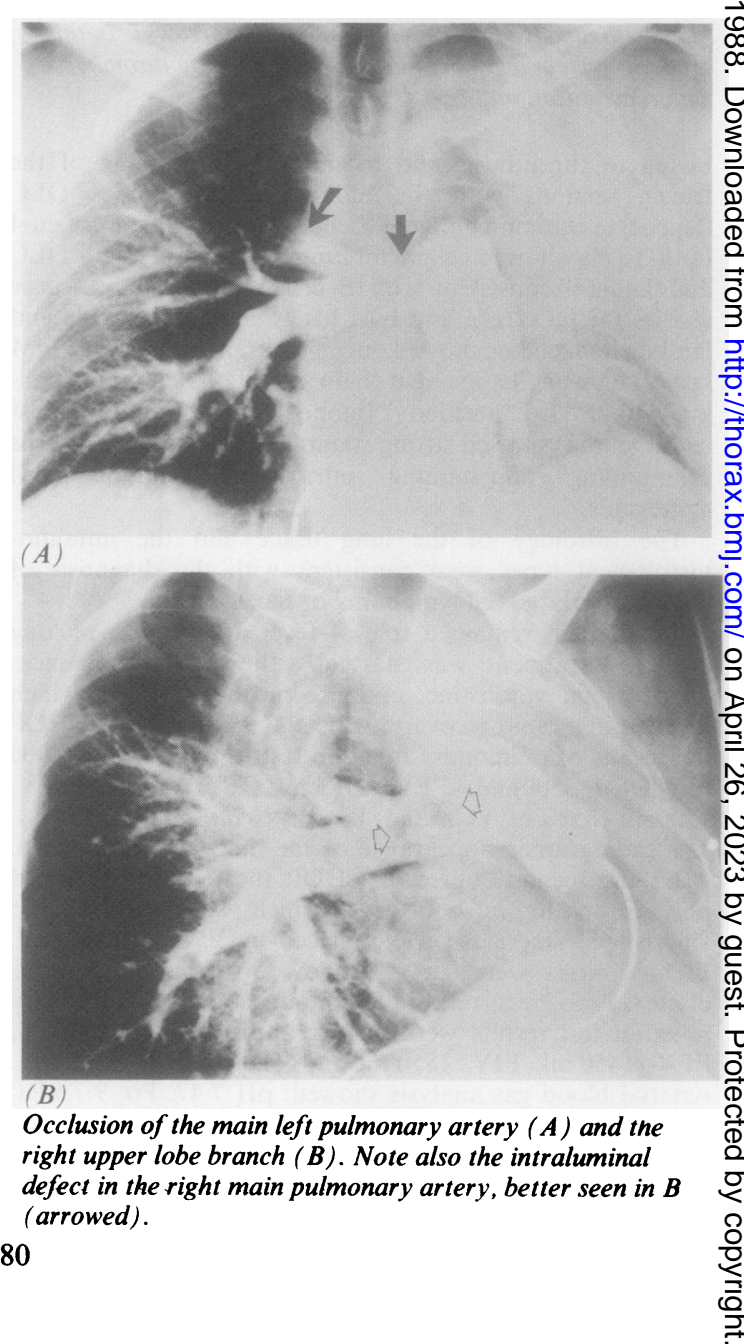




\section{Discussion}

Primary pulmonary artery sarcoma is a rare mesenchymal tumour. It appears to arise from intimal or subintimal tissue. Including this case, four of 83 reported histological diagnoses in primary pulmonary sarcoma have been malignant fibrous histiocytoma..$^{2-4}$ According to De Vita, malignant fibrous histiocytoma was the most common diagnosis in soft tissue sarcomas in recent series. ${ }^{5}$ The subdivision of the soft tissue tumours might be an imperfect classification, though it has previously had few therapeutic implications. ${ }^{6}$ These sarcomas almost always affect the pulmonary trunk and the left and right main pulmonary arteries. The pulmonary valve and the right ventricular outflow tract are affected less frequently. A multicentric origin is common. ${ }^{15}$ The tumour affects the lung by direct extension from the pulmonary vasculature and by distant metastases (pulmonary nodules) in about half of the cases. Distant metastases outside the lung are found less frequently..$^{\text {is }}$ Most cases were diagnosed at necropsy and only $\mathbf{4 0 \%}$, mainly recently reported cases, were suspected before surgery.

In most patients the primary diagnosis was pulmonary embolism, the duration of symptoms before the final diagnosis ranging from one month to 21 years (median duration 10 months). ${ }^{7}$ The peak incidence is from 50 to 55 years, with slightly more cases in females. The chest radiograph may show a hilar mass, usually unilateral and presenting on the lateral view as a segmental hilar shadow. ${ }^{8}$ Angiography (reported in 20-25 cases) might suggest the diagnosis, as does echocardiography, but most commonly the radiographic diagnosis, supported by perfusion-ventilation scans, is thromboembolism. Angiography may show either polypoid intraluminal masses protruding along the segmental arteries or simple occlusion, which cannot be differentiated from organised thrombi; in several cases thrombus formation has occurred on the tumour.

To differentiate tumour from embolism the following points are important. In our case and some others it was initially unilateral, with contralateral extension later (and almost always the pulmonary trunk was found to be affected at necropsy). This could be due to a multicentric origin, but could also have been by retrograde spread through change of flow after occlusion. A unilateral perfusion defect is seen in only $1 \%$ of cases of thromboembolism at angiography or in perfusion-ventilation scanning. A large embolus is unlikely if there is no associated history and if the contralateral pulmonary arteries are entirely normal. A neoplasm should be considered if a mass projects in the right outflow tract, though this can be diagnosed only by angiography. Another finding favouring tumour is failure of resolution of the perfusion defect at follow up, as resolution occurs in two out of three cases of thromboembolism within three months.

There is no effective treatment for pulmonary artery sarcoma. After surgery the longest reported survival has been 51 months. A poor and only temporary response has been reported in cases of malignant fibrous histiocytoma in other sites. $^{8}$

\section{References}

1 Baker PB, Goodwin RA. Pulmonary artery sarcomas. Arch Pathol Lab Med 1985;109:35-9.

2 Scully RE, Mark EJ, McNeely BU. Case records of the Massachusetts General Hospital (case 19-1985). N Engl J Med 1985;312:1242-52.

3 Bedrossian CWM, Verani R, Unger KM, et al. Pulmonary malignant fibrous histiocytoma. Chest 1979;75:186-9.

4 Misra DP, Sunderrajan EV, Rosenholtz MJ, Hurst DJ. Malignant fibrous histiocytoma in the lung masquerading as recurrent pulmonary thromboembolism. Cancer 1983;51:538-41.

5 De Vita VT, Hellman S, Rosenberg SA. Cancer: principles and practice of oncology. 2nd ed. Philadelphia: Lippincott Company, 1985:1262-3.

6 Kearny MM, Soule EH, Ivins JC. Malignant fibrous histiocytoma, a retrospective study of 167 cases. Cancer 1980;45:167-78.

7 Bleisch VR, Kraus FT. Polypoid sarcoma of the pulmonary trunk. Cancer 1980;46:314-24.

8 Moffat RE, Chang CHJ, Slaven JE. Roentgen considerations in primary pulmonary artery sarcoma. Radiology 1972;104:283-8. 